\title{
EQUICONVERGENCE THEOREMS FOR LAGUERRE SERIES
}

\author{
GEORGI E. KARADZHOV \\ Institute of Mathematics, Bulgarian Academy of Sciences \\ 1113 Sofia, Bulgaria
}

Abstract. The Szegö equiconvergence theorem for the Laguerre series is improved. In particular, a system of exact sufficient conditions is given.

1. Introduction and statement of the results. We shall consider the expansion of a function $f \in L_{\text {loc }}^{1}(0, \infty)$ in a Laguerre series: $f(y) \sim \sum_{n=0}^{\infty} a_{n} L_{n}(y, \alpha)$, where the coefficients $a_{n}$ are defined by

$$
\Gamma(\alpha+1)\left(\begin{array}{c}
n+\alpha \\
n
\end{array}\right) a_{n}=\int_{0}^{\infty} e^{-x} x^{\alpha} f(x) L_{n}(x, \alpha) d x, \quad \alpha>-1,
$$

and $L_{n}(x, \alpha)=(n !)^{-1} e^{x} x^{-\alpha}(d / d x)^{n}\left(e^{-x} x^{n+\alpha}\right)$ are the Laguerre polynomials. In [3] Szegö proves the following equiconvergence theorem:

TheOREM S. Let the integrals

$$
\int_{0}^{1} x^{\alpha}|f(x)| d x, \quad \int_{0}^{1} x^{\alpha / 2-1 / 4}|f(x)| d x
$$

exist. If

$$
\int_{n}^{\infty} e^{-x / 2} x^{\alpha / 2-13 / 12}|f(x)| d x=o\left(n^{-1 / 2}\right), \quad n \rightarrow \infty
$$

and if

$$
s_{n}(f, x)=\sum_{k=0}^{n} a_{k} L_{k}(x, \alpha)
$$


denotes the $n$-th partial sum of the Laguerre series of $f$, then

$$
s_{n}\left(f, y^{2}\right)-\frac{1}{\pi} \int_{y-c}^{y+c} f\left(x^{2}\right) \frac{\sin \sqrt{4 n}(x-y)}{x-y} d x=o(1), \quad n \rightarrow \infty,
$$

for every $y>c>0$, locally uniformly with respect to $y \in(c, \infty)$.

Moreover, (1.2) is valid if $\left(\mathrm{S}_{2}\right)$ is replaced by

$$
\int_{1}^{\infty} e^{-x / 2} x^{\alpha / 2-3 / 4}|f(x)| d x<\infty, \quad \int_{n}^{\infty} e^{-x} x^{\alpha-2}|f(x)|^{2} d x=o\left(n^{-3 / 2}\right) .
$$

The goal of this paper is to improve Theorem $\mathrm{S}$ as follows (see Theorems 1 and 2):

THEOREM 1. Let $h(x)=e^{-x / 2} x^{\alpha / 2-1 / 4} f(x), \alpha \geq-1 / 2$. If

$$
\begin{gathered}
\int_{0}^{1}|h(x)| d x<\infty, \\
\int_{1}^{\infty} x^{-1 / 2}|h(x)| d x<\infty, \\
\int a(\lambda, x)(1-x / \lambda)^{-1 / 4}|h(x)| d x=o\left(\lambda^{1 / 2}\right), \quad \lambda \rightarrow \infty,
\end{gathered}
$$

where $a(\lambda, x)$ is the characteristic function of the interval $\left(\lambda / 2, \lambda-\lambda^{1 / 3+\varepsilon}\right)$, and

$$
\int b(\lambda, x)|h(x)| d x=o\left(\lambda^{1 / 3}\right), \quad \lambda \rightarrow \infty,
$$

where $b(\lambda, x)$ is the characteristic function of $\left(\lambda-\lambda^{1 / 3+\varepsilon}, \lambda+\lambda^{1 / 3+\varepsilon}\right)$ for some $\varepsilon>0$, then the equiconvergence result (1.2) holds.

Rem ark 1 . If $\alpha \geq-1 / 2$, then the conditions $\left(\mathrm{H}_{1}\right)$ and $\left(\mathrm{S}_{1}\right)$ coincide and as is shown in [3], p. 248, they are exact. It is easy to see that $\left(\mathrm{S}_{2}\right)$ implies $\left(\mathrm{H}_{2}\right)-\left(\mathrm{H}_{4}\right)$. On the other hand, $\left(\mathrm{S}_{2}^{\prime}\right)$ implies $\left(\mathrm{H}_{2}\right),\left(\mathrm{H}_{3}\right)$ and

$$
\int b(\lambda, x)|h(x)| d x=o\left(\lambda^{1 / 6+\varepsilon}\right), \quad \lambda \rightarrow \infty,
$$

which is more restrictive than $\left(\mathrm{H}_{4}\right)$.

Remark 2. The condition $\left(\mathrm{H}_{4}\right)$ is also exact. Indeed, it is satisfied by the function $h(x)=x^{-\delta}$ for every $\delta>0$, but not for $\delta=0$. On the other hand, the Laguerre series of the function $f(x)=e^{x / 2} x^{-\alpha / 2+1 / 4}$ is divergent ([3], p. 267).

It turns out that for the functions $f(x)$ which are differentiable (or absolutely continuous) at infinity, we can improve the conditions $\left(\mathrm{H}_{2}\right)$ and $\left(\mathrm{H}_{3}\right)$ in such a way that they are satisfied by the function $f(x)=e^{x / 2} x^{-\alpha / 2+1 / 4-\delta}$ for every $\delta>0$. Namely, we have 
THEOREM 2. Let the function $g(x)=e^{-x^{2} / 2} x^{\alpha+1 / 2} f\left(x^{2}\right), \alpha \geq-1 / 2$, satisfy

$$
\int_{0}^{1}|g(x)| d x<\infty
$$

$$
\int_{N}^{\infty} x^{-2}\left|g^{\prime}(x)\right| d x<\infty \quad \text { for some large } N,
$$

$$
\begin{gathered}
\int a\left(\lambda, x^{2}\right)\left(1-x^{2} / \lambda\right)^{-1 / 4} x^{-2}\left|g^{\prime}(x)\right| d x=o(1), \quad \lambda \rightarrow+\infty \\
\int b\left(\lambda, x^{2}\right)|g(x)| d x=o\left(\lambda^{1 / 3}\right), \quad \lambda \rightarrow+\infty
\end{gathered}
$$

Then the equiconvergence relation (1.2) is valid.

EXAMPLE 1. The function $g(x)=x^{1-\delta}, 0<\delta<2$, has the properties $\left(\mathrm{H}_{1}\right)$, $\left(\mathrm{H}_{2}^{\prime}\right),\left(\mathrm{H}_{3}^{\prime}\right),\left(\mathrm{H}_{4}\right)$. The same is true for the functions $\left\{g(x): g(x)=O\left(x^{-1+\delta}\right)\right.$, $\left.x \rightarrow 0, \delta>0, g(x)=O\left(x^{1-\delta}\right), x \rightarrow \infty, g^{\prime}(x)=O\left(x^{1-\delta}\right), x \rightarrow \infty\right\}$. Therefore we have the following

Corollary 1. If $f \in L_{\mathrm{loc}}^{1}(0, \infty)$ and if $f(x)=O\left(x^{-\alpha / 2-3 / 4+\delta}\right), x \rightarrow 0$, and $f(x)=O\left(e^{x / 2} x^{-\alpha / 2+1 / 4-\delta}\right), f^{\prime}(x)=O\left(e^{x / 2} x^{-\alpha / 2+1 / 4-\delta}\right), x \rightarrow \infty$, where $\delta>0$, $\alpha \geq-1 / 2$, then the equiconvergence result (1.2) holds. (This is a system of exact sufficient conditions.)

Remark 3. Theorems 1 and 2 are also true for $-1<\alpha<-1 / 2$ if $\left(\mathrm{H}_{1}\right)$ is replaced by $\left(\mathrm{S}_{1}\right)$.

Let us explain briefly the main idea of the proof. We use the formula

$$
s_{n}\left(f, y^{2}\right)=2 \int_{0}^{\infty} e^{-x^{2} / 2+y^{2} / 2}(x / y)^{\alpha+1 / 2} f\left(x^{2}\right) e(4 n+4, x, y) d x
$$

where $e(\lambda, x, y)$ is the spectral function of the operator

$$
-d^{2} / d x^{2}+x^{2}+\left(\alpha^{2}-1 / 4\right) x^{-2}+2-2 \alpha,
$$

considered as a self-adjoint operator in $L^{2}(0, \infty)$. Namely,

$$
e(\lambda, x, y)=\left(e^{-x^{2} / 2-y^{2} / 2}\right) \frac{(x y)^{\alpha+1 / 2}}{\Gamma(\alpha+1)} \sum \frac{1}{\left(\begin{array}{c}
n+\alpha \\
n
\end{array}\right)} L_{n}\left(x^{2}, \alpha\right) L_{n}\left(y^{2}, \alpha\right) .
$$

Here, the sum is taken over all integers $n$ such that $0 \leq n \leq(\lambda-4) / 4$. Therefore, it suffices to know the uniform asymptotics of $e(\lambda, x, y)$ as $\lambda \rightarrow \infty$. To find it we consider the Laplace transform

$$
V(p, x, y)=\int_{0}^{\infty} e^{-\lambda p} d e(\lambda, x, y), \quad \operatorname{Re} p>0,
$$


and using its explicit expression ([3], p. 101), we derive the formula

$$
e(\lambda, x, y)=\frac{1}{2 \pi i} \int_{\varepsilon-i \pi / 4}^{\varepsilon+i \pi / 4} e^{\lambda p} V(p, x, y) H(\lambda, p) d p,
$$

where the function $s \rightarrow H(s, p)$ is 4-periodic, $H(s, p)=2 e^{(2-s) p}(\sinh 2 p)^{-1}$ if $0 \leq s \leq 4$. Next we apply the saddle-point method or the method of stationary phase.

For our purposes it is sufficient to consider the following cases: 1) $0<a_{1} \leq x \leq$ $2 A$; 2) $2 A \leq x \leq \sqrt{(1-\delta) \lambda} ; 3) \sqrt{(1-\delta) \lambda} \leq x \leq \sqrt{(1+\delta) \lambda}$; 4$) x \geq \sqrt{(1+\delta) \lambda}$, provided that $0<a_{2} \leq y \leq A$.

Theorem 3 (the case $0<a_{1} \leq x \leq 2 A, 0<a_{2} \leq y \leq A$ ). We have the uniform asymptotics

$$
e(\lambda, x, y)=\frac{1}{2 \pi} \frac{\sin \sqrt{\lambda}(x-y)}{x-y}+\frac{c_{\alpha}}{2 \pi} \frac{\sin \sqrt{\lambda}(x+y)}{x+y}+O\left(\lambda^{-1 / 2}\right),
$$

$\lambda \rightarrow \infty$, for some constant $c_{\alpha}$.

It will be convenient to consider also the function

$$
E(\lambda, x, y)=e(\lambda, \sqrt{\lambda} x, \sqrt{\lambda} y) .
$$

Theorem 4 (the case $4 A^{2} / \lambda \leq x^{2} \leq 1-\delta, 0<a_{2} \leq \sqrt{\lambda} y \leq A$ ). For every small $\delta>0$ we have the uniform asymptotics

$$
\begin{aligned}
E(\lambda, x, y) & =F(\lambda, x, y)+c_{\alpha} F(\lambda, x,-y), \\
\left(1-x^{2}\right)^{1 / 4} F(\lambda, x, y)= & \lambda^{-1 / 2} \sum_{j=1}^{4} b_{j}(\lambda, x, y) \exp \left(i \lambda \psi_{j}(x, y)\right) \\
& +x^{-1} O\left(\lambda^{-3 / 2}\right), \quad \lambda \rightarrow+\infty,
\end{aligned}
$$

where $\left|b_{j}\right| \leq c x^{-1},\left|\partial_{x} b_{j}\right| \leq c x^{-2}$ and

$$
\left|\partial_{x} \psi_{j}(x, y)\right| \geq c x, \quad 1 \leq j \leq 4,
$$

for some constant $c>0$.

Corollary 2. If $2 A \leq x \leq \lambda / 2,0<a_{2} \leq y \leq A$ then the uniform estimate $|e(\lambda, x, y)| \leq c x^{-1}$ is valid.

TheOREM 5 (the case $\left.1-\delta \leq x^{2} \leq 1+\delta, 0<a_{2} \leq \sqrt{\lambda} y \leq A\right)$. There exists a positive number $\delta$ such that we have the uniform asymptotics (1.8) where

$$
F(\lambda, x, y)=\lambda^{-1 / 3} \sum_{k \geq 0}\left(\alpha_{0 k}(\lambda, x, y) \lambda^{-k}+\alpha_{1 k}(\lambda, x, y) \lambda^{-k-1 / 3}\right), \quad \lambda \rightarrow+\infty,
$$

and

$$
\begin{gathered}
\alpha_{j k}=\left(a_{j k} e^{\lambda A}+b_{j k} e^{\lambda \bar{A}} \mathrm{Ai}^{(j)}\left(\lambda^{2 / 3} B\right),\right. \\
\left|a_{j k}\right|+\left|\partial_{x} a_{j k}\right| \leq c, \quad\left|b_{j k}\right|+\left|\partial_{x} b_{j k}\right| \leq c, \quad j=0,1 .
\end{gathered}
$$


Here $\operatorname{Ai}(s)=(2 \pi)^{-1} \int \exp \left(i\left(s t+t^{3} / 3\right)\right) d t$ is the Airy function and

$$
\begin{gathered}
A=A(x, y)=\frac{1}{2}\left(\varphi\left(p_{+}, x, y\right)+\varphi\left(p_{-}, x, y\right)\right), \\
B=B(x, y)=\left(\frac{3}{4}\left(\varphi\left(p_{+}, x, y\right)-\varphi\left(p_{-}, x, y\right)\right)\right)^{2 / 3},
\end{gathered}
$$

where

$$
\varphi(p, x, y)=p-\frac{1}{2}\left(x^{2}+y^{2}\right) \operatorname{coth} 2 p+x y(\sinh 2 p)^{-1}
$$

and

$$
\begin{array}{rlr}
\text { (1.16) } & p_{ \pm}=i t_{ \pm}, \quad \cos 2 t_{ \pm}=-x y \pm\left(\left(1-x^{2}\right)\left(1-y^{2}\right)\right)^{1 / 2}, \\
0<t_{ \pm}<\pi / 2 & \text { if } x<1, \\
\text { (1.17) } & p_{ \pm}= \pm \varepsilon+i t, \quad \cosh 2 \varepsilon=x, \quad \varepsilon>0, & \cos 2 t=-y, \\
0<t<\pi / 2 & \text { if } x>1 .
\end{array}
$$

Remark 4 . The smooth functions $A(x, y), B(x, y)$ satisfy

$$
\begin{gathered}
B(x, \pm y)>0 \quad \text { if } x>1, \quad B(x, \pm y)<0 \quad \text { if } x<1 \\
B(x, \pm y)=-2^{1 / 3}(1-x)(1+O(1-x)) \quad \text { as } x \rightarrow 1 \\
\operatorname{Re} A(x, \pm y)=0 .
\end{gathered}
$$

Corollary 3. If $1-\delta<x^{2}<1-\lambda^{-2 / 3+\varepsilon}, 0<a_{2} \leq \sqrt{\lambda} y \leq A$ for some $\varepsilon>0$, then we have the uniform asymptotics (1.8) where

$$
F(\lambda, x, y)=\lambda^{-1 / 2}\left(1-x^{2}\right)^{-1 / 4} \sum_{j=1}^{4} \sum_{k \geq 0} a_{k j}\left(\lambda^{2 / 3}\left(1-x^{2}\right)\right)^{-3 k / 2} \exp \left(i \lambda \psi_{j}\right)
$$

$\lambda \rightarrow \infty$, the functions $\psi_{j}$ satisfy $(1.10)$ and

$$
\left|a_{k j}(\lambda, x, y)\right|+\left|\partial_{x} a_{k j}(\lambda, x, y)\right| \leq c .
$$

Theorem 6 (the case $x^{2} \geq 1+\delta, 0<a_{2} \leq \sqrt{\lambda} y \leq A$ ). For every small $\delta>0$ the uniform estimate $|E(\lambda, x, y)| \leq c \lambda^{-1 / 2} \exp \left(-\frac{1}{2} \lambda \delta \sqrt{x^{2}-1}\right)$ holds.

Theorems 5 and 6 imply the following

Corollary 4. If $x^{2} \geq \lambda+\lambda^{1 / 3+\varepsilon}, \varepsilon>0$, and $0<a_{2} \leq y \leq A$ then we have the uniform estimate

$$
|e(\lambda, x, y)| \leq c \lambda^{-1 / 3} \exp \left(-c \lambda^{1 / 3}\left(x^{2} / \lambda-1\right)^{1 / 2}\right) .
$$

\section{Proof of the equiconvergence theorems}

Proof of Theorem 1. Let $g(x)=e^{-x^{2} / 2} x^{\alpha+1 / 2} f\left(x^{2}\right)$. As in the proof of Theorem S ([3], p. 264), it suffices to establish a uniform estimate of the kind

$$
R_{n}\left(f, y^{2}\right)=O(1)\left(\int_{0}^{1}|g(x)| d x+\int_{1}^{\infty} x^{-1}|g(x)| d x\right)+o(1)
$$


$n \rightarrow \infty$, where $0<c<a_{2} \leq y \leq A$ and

$$
R_{n}\left(f, y^{2}\right)=s_{n}\left(f, y^{2}\right)-\frac{1}{\pi} \int_{y-c}^{y+c} f\left(x^{2}\right) \frac{\sin \sqrt{4 n}(x-y)}{x-y} d x .
$$

Using (1.3), (1.7) and the Riemann-Lebesgue lemma, we have

$$
R_{n}\left(f, y^{2}\right)=\left(\int_{0}^{a_{1}}+\int_{2 A}^{\infty}\right) g(x) e(4 n+4, x, y) d x+o(1),
$$

$n \rightarrow \infty$, where $0<a_{2} \leq y \leq A$ and $a_{1}=a_{2}-c$. Since

$$
\int_{0}^{a_{1}}|g(x) e(4 n+4, x, y)| d x=O(1) \int_{0}^{1}|g(x)| d x \quad \text { if } \alpha \geq-1 / 2
$$

(see [3], p. 264), it remains to estimate the integrals

$$
K_{j}(\lambda, y)=\int a_{j}\left(\lambda, x^{2}\right) g(x) e(\lambda, x, y) d x, \quad 1 \leq j \leq 4,
$$

uniformly with respect to $y \in\left[a_{2}, A\right], a_{2}>0$, where $x \rightarrow a_{j}(\lambda, x)$ is the characteristic function of the interval $I_{j}, 1 \leq j \leq 4$, and $I_{1}=\left(4 A^{2}, \lambda / 2\right), I_{2}=$ $\left(\lambda / 2, \lambda-\lambda^{1 / 3+\varepsilon}\right), I_{3}=\left(\lambda-\lambda^{1 / 3+\varepsilon}, \lambda+\lambda^{1 / 3+\varepsilon}\right), I_{4}=\left(\lambda+\lambda^{1 / 3+\varepsilon}, \infty\right)$. To estimate the integral $K_{1}$ we apply Corollary 2 to get

$$
K_{1}(\lambda, y)=O(1) \int_{1}^{\infty} x^{-1}|g(x)| d x
$$

Further, Theorem 4 and Corollary 3 imply the estimate $\left|e(\lambda, x, y) a_{2}\left(\lambda, x^{2}\right)\right| \leq$ $c^{-1 / 2}\left(1-x^{2} / \lambda\right)^{-1 / 4}$, hence $\left(\mathrm{H}_{3}\right)$ gives

$$
K_{2}(\lambda, y)=o(1), \quad \lambda \rightarrow \infty .
$$

Theorem 5 and $\left(\mathrm{H}_{4}\right)$ show that

$$
K_{3}(\lambda, y)=o(1), \quad \lambda \rightarrow \infty .
$$

Corollary 4 yields

$$
\begin{aligned}
\left|K_{4}(\lambda, y)\right| \leq c\left(\lambda^{3} e^{-c \lambda^{\varepsilon / 2}}\right. & \int b_{1}\left(\lambda, x^{2}\right)|g(x)| x^{-3} d x \\
& \left.+\lambda^{-1 / 3} \int b_{2}\left(\lambda, x^{2}\right)|g(x)| \exp \left(-c x^{1 / 2}\right) d x\right),
\end{aligned}
$$

where $x \rightarrow b_{1}(\lambda, x)$ is the characteristic function of $\left(\lambda+\lambda^{1 / 3+\varepsilon}, \lambda^{2}\right)$ and $b_{1}+b_{2}=$ $a_{4}$. Therefore $(2.9)$ and $\left(\mathrm{H}_{2}\right)$ give

$$
K_{4}(\lambda, y)=o(1), \quad \lambda \rightarrow \infty .
$$

Evidently, (2.1) follows from (2.3)-(2.10). Theorem 1 is proved.

Proof of Theorem 2. We use again (2.2)-(2.4). Note first that $\left(\mathrm{H}_{3}^{\prime}\right)$ and $\left(\mathrm{H}_{4}\right)$ imply

$$
g(x)=O\left(x^{5 / 3}\right), \quad x \rightarrow \infty .
$$


Indeed, let $x_{n} \rightarrow \infty$ and $x_{n}^{2}=\lambda_{n}+\lambda_{n}^{1 / 3}$. Then $\lambda_{n}-\lambda_{n}^{1 / 3}<x_{n}^{2}-x_{n}^{2 / 3}$ for large $n$. Using $\left(\mathrm{H}_{4}\right)$ and the mean-value theorem, we find $y_{n}$ such that $x_{n}^{2}-x_{n}^{2 / 3}<y_{n}^{2}<x_{n}^{2}$ and $g\left(y_{n}\right)=O\left(x_{n}^{5 / 3}\right), n \rightarrow \infty$. Further, choose $\lambda_{n}$ so that $x_{n}^{2}=\lambda_{n}-\lambda_{n}^{1 / 3}$. Since $g\left(x_{n}\right)-g\left(y_{n}\right)=\int_{y_{n}}^{x_{n}} g^{\prime}(x) d x$ and

$$
\begin{aligned}
& \left|g\left(x_{n}\right)-g\left(y_{n}\right)\right| \leq x_{n}^{2} \int_{y_{n}}^{x_{n}}\left(1-x^{2} / \lambda_{n}\right)^{1 / 4-1 / 4} x^{-2}\left|g^{\prime}(x)\right| d x, \\
& 1-x^{2} / \lambda_{n}<1-y_{n}^{2} / \lambda_{n}<2 \lambda_{n}^{-2 / 3}<2 x_{n}^{-1 / 3} \quad \text { if } x_{n}>y_{n},
\end{aligned}
$$

we get from $\left(\mathrm{H}_{3}^{\prime}\right)$ the estimate $\left|g\left(x_{n}\right)-g\left(y_{n}\right)\right| \leq c x_{n}^{5 / 3}$. Thus, (2.11) follows.

Now as in the proof of Theorem 1 it is sufficient to see that

$$
\begin{aligned}
R_{n}\left(f, y^{2}\right)=O(1)\left(\int_{0}^{1}|g(x)| d x\right. & +\int_{1}^{\infty} x^{-3}|g(x)| d x \\
& \left.+\int_{N}^{\infty} x^{-2}\left|g^{\prime}(x)\right| d x\right)+o(1), \quad n \rightarrow \infty
\end{aligned}
$$

uniformly in $\left[a_{2}, A\right], a_{2}>0$. To this end we shall estimate the integrals $K_{j}$, $1 \leq j \leq 4$, from $(2.5)$. It is clear that $(2.8)-(2.10)$ remain valid. To estimate $K_{1}$ and $K_{2}$ we consider the formulas

$$
\begin{aligned}
B_{j}(\lambda, y) & =K_{j}(\lambda, \sqrt{\lambda} y) \\
& =\sqrt{\lambda} \int a_{j}\left(\lambda, \lambda x^{2}\right) g(\sqrt{\lambda} x) E(\lambda, x, y) d x, \quad j=1,2 .
\end{aligned}
$$

Using an appropriate partition of unity in the integral (1.3), we can suppose that $g(2 A)=0$. In the integral $B_{1}$, integration by parts with the help of Theorem 4 gives

$$
K_{1}(\lambda, y)=O(1)\left(\int_{1}^{\infty} x^{-3}|g(x)| d x+\int_{N}^{\infty} x^{-2}\left|g^{\prime}(x)\right| d x\right) .
$$

In the integral $B_{2}$ we integrate by parts, using Theorem 4 (if $1 / 2<x^{2}<1-\delta$ ) and Corollary 2 (if $1-\delta<x^{2}<1-\lambda^{-2 / 3+\varepsilon}$ ). Taking into account (2.11), we get

$$
\begin{aligned}
B_{2}(\lambda, y)=O(1)\left(\int_{1}^{\infty} x^{-3}|g(x)| d x+\int_{N}^{\infty} x^{-2}\left|g^{\prime}(x)\right|\right. & d x \\
& \left.+\lambda^{-\varepsilon / 4}+B(\lambda, y)\right)
\end{aligned}
$$

for small $\varepsilon>0$, where

$$
\begin{aligned}
& B(\lambda, y)=C(\lambda, y)+c_{\alpha} C(\lambda,-y) \\
& C(\lambda, y)=\lambda^{-1} \sum_{j=1}^{4} \sum_{k=0}^{M} \int a_{2}\left(\lambda, \lambda x^{2}\right) e^{i \lambda \psi_{j}} \frac{\partial}{\partial x} q(\lambda, x) d x,
\end{aligned}
$$




$$
q(\lambda, x)=g(\sqrt{\lambda} x) a_{k j}(\lambda, x)\left(1-x^{2}\right)^{-1 / 4}\left(\partial_{x} \psi_{j}\right)^{-1}\left(\lambda^{2 / 3}\left(1-x^{2}\right)\right)^{-3 k / 2}
$$

and $M=M(\varepsilon)$ is large enough. Since the functions $a_{k j}(\lambda, x),\left(\partial_{x} \psi_{j}\right)^{-1}$ and their derivatives with respect to $x$ are bounded when $1 / 2<x^{2}<1$, it is sufficient to estimate the integrals

$$
\begin{aligned}
& C_{1}(\lambda, y)=\lambda^{-1} \int a_{2}\left(\lambda, \lambda x^{2}\right)\left(1-x^{2}\right)^{-5 / 4}|g(\sqrt{\lambda} x)| d x, \\
& C_{2}(\lambda, y)=\lambda^{-1 / 2} \int a_{2}\left(\lambda, \lambda x^{2}\right)\left(1-x^{2}\right)^{-1 / 4}\left|g^{\prime}(\sqrt{\lambda} x)\right| d x .
\end{aligned}
$$

By virtue of (2.11), we have

$$
C_{1}(\lambda, y) \leq c \int_{x^{2}<1}\left(1-x^{2}\right)^{-1+\delta} d x \lambda^{-\varepsilon / 8} \quad \text { if } \delta=\frac{3 \varepsilon}{8(2-3 \varepsilon)}
$$

where $\varepsilon>0$ is small enough. On the other hand,

$$
C_{2}(\lambda, y) \leq c \int a_{2}\left(\lambda, x^{2}\right)\left(1-x^{2} / \lambda\right)^{-1 / 4} x^{-2}\left|g^{\prime}(x)\right| d x .
$$

It is not hard to see that $(2.13),(2.15)-(2.22)$ and $\left(\mathrm{H}_{3}^{\prime}\right)$ yield the estimate

$$
K_{2}(\lambda, y)=O(1)\left(\int_{1}^{\infty} x^{-3}|g(x)| d x+\int_{N}^{\infty} x^{-2}\left|g^{\prime}(x)\right| d x\right)+o(1),
$$

$\lambda \rightarrow \infty$. Consequently, (2.12) follows from (2.3), (2.4), (2.8)-(2.10) and (2.14), (2.23). Theorem 2 is proved.

\section{Proof of the asymptotics for the spectral function}

Proof of Theorem 3. First we prove the formula (1.6). According to Theorem 5.1 of [3], we can write

$$
V(p, x, y)=\frac{1}{2}(x y)^{1 / 2} e^{2 p(\alpha-1)}(\sinh 2 p)^{-1} e^{(1 / 2)\left(x^{2}+y^{2}\right) \operatorname{coth} 2 p} i^{-\alpha} J_{\alpha}\left(\frac{i x y}{\sinh 2 p}\right)
$$

if $\operatorname{Re} p>0$. Notice that

$$
V(p+i k \pi / 2, x, y)=V(p, x, y) .
$$

From (1.4), (1.5) it follows that

$$
V(p, x, y)=p \int_{0}^{\infty} e^{-\lambda p} e(\lambda, x, y) d \lambda, \quad \operatorname{Re} p>0 .
$$

We want to apply the inverse Laplace formula. Since the function $\lambda \rightarrow e(\lambda, x, y)$ is only right-continuous, it is convenient to consider the Steklov average:

$$
e_{h}(\lambda, x, y)=\frac{1}{h} \int_{0}^{h} e(\lambda+\mu, x, y) d \mu, \quad h>0 .
$$


Evidently $e_{h}(\lambda, x, y) \rightarrow e(\lambda, x, y)$ as $h \rightarrow+0$ for every fixed $(\lambda, x, y)$ and (3.2) can be rewritten as follows:

$$
\int_{0}^{\infty} e^{-\lambda p} e_{h}(\lambda, x, y) d \lambda=\frac{e^{h p}-1}{h^{2}} \cdot \frac{V(p, x, y)}{p^{2}}, \quad h>0, \operatorname{Re} p>0 .
$$

Hence the inverse Laplace formula gives

$$
e_{h}(\lambda, x, y)=\frac{1}{2 \pi i} \int_{\varepsilon-i \infty}^{\varepsilon+i \infty} e^{\lambda p} \frac{e^{h p}-1}{h^{2}} \cdot \frac{V(p, x, y)}{p^{2}} d p, \quad \varepsilon>0 .
$$

Now the periodicity relation (3.1) and the Weierstrass theorem show that

$$
e_{h}(\lambda, x, y)=\frac{1}{2 \pi i} \int_{\varepsilon-i \pi / 4}^{\varepsilon+i \pi / 4} e^{\lambda p} V(p, x, y) \frac{g(h, p)-g(0, p)}{h} d p
$$

where $g(s, p)=e^{p s} f(\lambda+s, p)$ and $f(s, p)=\sum e^{i s k \pi / 2}(p+i k \pi / 2)^{-2}$. The function $s \rightarrow f(s, p)$ is continuous, 4-periodic and

$$
f(s, p)=4 e^{(2-s) p}(\operatorname{coth} 2 p+s / 2-1)(\sinh 2 p)^{-1}
$$

for $0 \leq s<4, \operatorname{Re} p>0$. In particular, $\lim _{h \rightarrow+0} h^{-1}(g(h, p)-g(0, p))=H(\lambda, p)$ and using the Lebesgue theorem we get (1.6) from (3.3). Notice also that

$$
p \rightarrow e^{\lambda p} V(p, x, y) H(\lambda, p) \quad \text { is } \quad i \pi / 2 \text {-periodic . }
$$

This allows us to write

$$
\begin{aligned}
e(\lambda, x, y)= & \frac{1}{2 \pi i}\left(\int_{\varepsilon-i \pi / 4}^{\varepsilon+i \pi / 4} e^{\lambda p} V(p, x, y) H(\lambda, p) \chi_{1}(p) d p\right. \\
& \left.+\int_{\varepsilon+i 0}^{\varepsilon+i \pi / 2} e^{\lambda p} V(p, x, y) H(\lambda, p) \chi_{2}(p) d p\right)
\end{aligned}
$$

where $\chi_{1}(p)+\chi_{2}(p)=1$ on the interval $\{p=\varepsilon+i t:|t| \leq \pi / 4\}$ and $\operatorname{supp} \chi_{1}(p) \subset$ $\{p=\varepsilon+i t:|t| \leq \gamma<\pi / 4\}, \chi_{1}(\varepsilon+i t)=1$ if $|t| \leq \gamma / 2$ and the function $\chi_{2}(p)$ is $i \pi / 2$-periodic. Thus

$$
e(\lambda, x, y)=\frac{1}{2 \pi i} \int_{S} e^{\lambda p} V(p, x, y) H(\lambda, p) \chi(p) d p
$$

where $S=(\varepsilon-i \pi / 2, \varepsilon+i \pi / 2)$ and $\chi \in C_{0}^{\infty}(S), \chi(\varepsilon+i t)=1$ if $|t| \leq \pi / 8$.

We shall now find an appropriate form of $V(p, x, y)$, separating the oscillating part. Using the formulas (1), p. 74, (6), p. 75 and (3), (4), p. 168 of [4], we can write

$$
J_{\alpha}(z)=z^{-1 / 2}\left(e^{i z} c_{\alpha}^{+} f(-z)+e^{-i z} c_{\alpha}^{-} f(z)\right) \quad \text { if } \alpha \geq-1 / 2
$$


where

(3.6) $f(z)= \begin{cases}\frac{1}{2}\left(\frac{2}{\pi}\right)^{1 / 2} \frac{1}{\Gamma(\alpha+1 / 2)} \int_{0}^{\infty} e^{-u} u^{\alpha-1 / 2}\left(1-\frac{i u}{2 z}\right)^{-1 / 2} d u & \text { if } \alpha>-1 / 2, \\ \frac{1}{2}\left(\frac{2}{\pi}\right)^{1 / 2} & \text { if } \alpha=-1 / 2,\end{cases}$

is a holomorphic function for $\operatorname{Re} z \neq 0$. Here $c_{\alpha}^{-}=e^{i(\pi / 2)(\alpha+1 / 2)}$. Therefore

$$
\begin{aligned}
V(p, x, y)=(\sinh 2 p)^{-1 / 2} e^{-(1 / 2)\left(x^{2}+y^{2}\right) \operatorname{coth} 2 p} & \left(e^{x y / \sinh 2 p} a(p, x y)\right. \\
& \left.+e^{-x y / \sinh 2 p} c_{\alpha} a(p,-x y)\right)
\end{aligned}
$$

where $c_{\alpha}=e^{-i(\pi / 2)(\alpha+1 / 2)} c_{\alpha}^{+}$and

$$
a(p, x y)=\frac{1}{2} e^{2 p(\alpha-1)} f(i x y / \sinh 2 p) .
$$

Since $-\frac{1}{2}\left(x^{2}+y^{2}\right) \operatorname{coth} 2 p+x y / \sinh 2 p=-(x-y)^{2} /(4 p)+s(p, x, y)$ and $s(0, x, y)$ $=0$, we have the representation

$$
V(p, x, y)=p^{-1 / 2}\left(e^{-(x-y)^{2} /(4 p)} b(p, x, y)+e^{-(x+y)^{2} /(4 p)} c_{\alpha} b(p, x,-y)\right)
$$

where $b(0, x, \pm y)=1 /(4 \sqrt{\pi})$. Further, we have the equality

$$
\frac{1}{2 \sqrt{\pi p}} e^{-(x-y)^{2} /(4 p)}=\frac{1}{2 \pi i(x-y)} \int 2 \xi p e^{-\xi^{2} p+i(x-y) \xi} d \xi, \quad \operatorname{Re} p>0
$$

therefore

$$
V(p, x, y)=W(p, x, y)+c_{\alpha} W(p, x,-y)
$$

where

(3.10) $W(p, x, y)=\frac{p}{x-y} a(p, x, y) \int \xi e^{-\xi^{2} p+i(x-y) \xi} d \xi, \quad \operatorname{Re} p>0$,

(3.11) $\quad a(0, x, \pm y)=1 /(2 \pi i)$.

Now (3.5), (3.9) and (3.10) show that

$$
E(\lambda, x, y)=F(\lambda, x, y)+c_{\alpha} F(\lambda, x,-y)
$$

where

$$
F(\lambda, x, y)=\frac{\sqrt{\lambda}}{x-y} \int e^{i \lambda \psi(t, \xi, x, y)} q(t, \xi, \lambda, x, y) d t d \xi
$$

is an oscillating integral with respect to $\xi$, and

$$
\begin{aligned}
q(t, \xi, \lambda, x, y) & =\frac{\xi}{2 \pi} a(i t, \sqrt{\lambda} x, \sqrt{\lambda} y) H(\lambda, i t) i t \chi(i t), \\
\psi(t, \xi, x, y) & =\left(1-\xi^{2}\right) t+(x-y) \xi .
\end{aligned}
$$

Notice that $a_{1} \leq \sqrt{\lambda} x \leq 2 A, a_{2} \leq \sqrt{\lambda} y \leq A$ and from (3.14) and (3.6)-(3.10) it follows that

$$
\left|\partial_{t}^{k} q\right| \leq C_{k}|\xi|
$$


Since $\left|\partial_{t} \psi\right| \geq c \xi^{2}$ if $\xi^{2}$ is large enough, we can integrate by parts in the integral (3.13) to get

$$
F(\lambda, x, y) \sim \frac{\sqrt{\lambda}}{x-y} \int e^{i \lambda \psi(t, \xi, x, y)} \kappa(\xi) q(t, \xi, \lambda, x, y) d t d \xi
$$

where $\kappa \in C_{0}^{\infty}(\mathbb{R})$ is an even cut-off function and the equivalence relation " $A(\lambda, x, y) \sim B(\lambda, x, y)$ " here means that $A(\lambda, x, y)-B(\lambda, x, y)=O\left(\lambda^{-\infty}\right)$, uniformly with respect to $x, y$. Applying the method of stationary phase to the integral (3.17), we derive the uniform asymptotics

$$
F(\lambda, x, y)=\lambda^{-1 / 2}(2 \pi(x-y))^{-1} \sin \lambda(x-y)+O\left(\lambda^{-1 / 2}\right), \quad \lambda \rightarrow \infty .
$$

Together with (3.12), (3.13), this gives (1.7). Theorem 3 is proved.

Proof of Theorem 4. We start from the formulas (3.5) and (3.7). Now we use the equality

$$
\int e^{-\xi^{2}(\sinh 2 p) / 2+i(x-y) \xi} \xi d \xi=\sqrt{2 \pi} i(x-y)(\sinh 2 p)^{-3 / 2} g(p),
$$

$\operatorname{Re} p>0$, where

$$
g(p)=\exp \left(\frac{x^{2}+y^{2}}{2} \tanh p-\frac{x^{2}+y^{2}}{2} \operatorname{coth} 2 p+\frac{x y}{\sinh 2 p}\right) .
$$

Therefore we have again the representation (3.9), where

$$
\begin{aligned}
W(p, x, y) & =(x-y)^{-1}(\sinh 2 p) a(p, x, y) \int \xi \exp (\psi(p, \xi)) d \xi, \\
\psi(p, \xi) & =-\xi^{2}(\sinh 2 p) / 2+i(x-y) \xi-\left(x^{2}+y^{2}\right)(\tanh p) / 2, \\
a(p, x, y) & =(\exp (2 p(\alpha-1)-i \pi(\alpha+1 / 2) / 2)) /(2 i \sqrt{2 \pi}) f(i x y / \sinh 2 p) .
\end{aligned}
$$

Analogously to (3.12)-(3.17) we obtain again (3.12), (3.17), where the phase function $\psi$ now has the form

$$
\psi(t, \xi, x, y)=t-\frac{\xi^{2}}{2} \sin 2 t-\frac{x^{2}+y^{2}}{2} \tan t+(x-y) \xi
$$

and

$$
q(t, \xi, \lambda, x, y)=(\xi /(2 \pi)) a(i t, \sqrt{\lambda} x, \sqrt{\lambda} y) H(\lambda, i t) i(\sin 2 t) \chi(i t),
$$

so the estimate (3.16) is valid.

Applying the method of stationary phase, we see that the function (3.18) has four nondegenerate critical points: $\left(t_{ \pm}, \xi_{ \pm}\right)$and $\left(-t_{ \pm},-\xi_{ \pm}\right)$, where $\cos 2 t_{ \pm}=$ $x y \pm \omega, \omega=\left(\left(1-x^{2}\right)\left(1-y^{2}\right)\right)^{1 / 2}, \xi_{ \pm} \sin 2 t_{ \pm}=x-y$. In addition, for the Hessian $\psi^{\prime \prime}$ we have $\operatorname{det} \psi^{\prime \prime}\left(t_{ \pm}, \xi_{ \pm}\right)= \pm 4 \omega$. Thus the method of stationary phase yields the asymptotics $(1.9)$, where $\psi_{1}(x, y)=\psi\left(t_{+}, \xi_{+}, x, y\right), \psi_{2}(x, y)=\left(t_{-}, \xi_{-}, x, y\right)$, $\psi_{3}=-\psi_{1}, \psi_{4}=-\psi_{2}$ and $b_{k}(\lambda, x, y)=(x-y)^{-1} a_{k}(\lambda, x, y),\left|\partial_{x} a_{k}\right| \leq c x^{-1}$, $1 \leq k \leq 4$. Theorem 4 is proved. 
Proof of Theorem 5. Starting from the formula (1.6), we use the periodicity property (3.4) and obtain the representation

$$
e(\lambda, x, y)=\frac{1}{4 \pi i} \int_{S} e^{\lambda p} V(p, x, y) H(\lambda, p) d p, \quad \operatorname{Re} p>0,
$$

where $S=(\varepsilon-i \pi / 2, \varepsilon+i \pi / 2)$. Further, (3.7), (3.8) show that

$$
E(\lambda, x, y)=F(\lambda, x, y)+c_{\alpha} F(\lambda, x,-y)
$$

where

$$
F(\lambda, x, y)=\int_{S} e^{\lambda \varphi(p, x, y)} q(p, \lambda, x, y) d p
$$

$\varphi$ is given by (1.15) and

$$
q(p, \lambda, x, y)=\frac{e^{2 p(\alpha-1)}}{8 \pi i}(\sinh 2 p)^{-1 / 2} f(\lambda i x y / \sinh 2 p) H(\lambda, p) .
$$

To find the uniform asymptotics of the integral (3.20) as $\lambda \rightarrow \infty$, we shall apply the saddle-point method. Since $a_{2} \leq \sqrt{\lambda} y \leq A$ the phase function $p \rightarrow \varphi(p, x, y)$ has critical points $p_{ \pm}$and $\bar{p}_{ \pm}$, where $p_{ \pm}$are given by (1.16), (1.17). If $x=1$, then $p_{ \pm}=p_{0}=i t_{0}$ where $\cos 2 t_{0}=y$ and $0<t_{0}<\pi / 2$. Hence, the critical points $p_{0}$ and $\bar{p}_{0}$ are degenerate and $\left(\partial^{3} \varphi / \partial p^{3}\right)(p, x, y)=8,\left(\partial^{2} \varphi / \partial p \partial x\right)(p, x, y)=-2$ if $p=p_{0}$ or $p=\bar{p}_{0}$. Since $\left|x^{2}-1\right|<\delta$, we can choose $\delta>0$ so that $0<|\operatorname{Im} p|<\pi / 2$ for all the critical points. Consequently, the integrand in (3.20) is holomorphic near the critical points. On the other hand, according to Lemma 2.3 of $[1$, p. 343], we can find a holomorphic change of variables $p=p(z, x, y)$ in a neighborhood of the points $z=0, x=1$ such that

$$
\varphi(p(z, x, y), x, y)=A(x, y)-B(x, y) z+z^{3} / 3, \quad p(0,1, y)=p_{0} .
$$

Note also that (3.22) and (1.15) imply

$$
\begin{aligned}
\varphi(\overline{p(\bar{z}, x, y)}, x, y) & =\overline{A(x, y)}-B(x, y) z+z^{3} / 3, \\
\overline{p(0,1, y)} & =\overline{p_{0}},
\end{aligned}
$$

and

$$
\overline{p( \pm \overline{\sqrt{B}}, x, y)}= \begin{cases}\bar{p}_{ \pm} & \text {if } x>1, \\ \bar{p}_{\mp} & \text { if } x<1 .\end{cases}
$$

To use the holomorphic change of variables (3.22), (3.23) in the integral (3.20), we shall prove first that

$$
F(\lambda, x, y) \sim \int_{\gamma} e^{\lambda \varphi(p, x, y)} q(p, \lambda, x, y), \quad \gamma=\gamma_{1} \cup \gamma_{2},
$$

where $\gamma_{1}$ is the segment $\left(\varepsilon+i\left(t_{0}-2 \varepsilon\right), \varepsilon+i\left(t_{0}+2 \varepsilon\right)\right)$ and $\gamma_{2}$ the segment $(\varepsilon-$ $\left.i\left(t_{0}+2 \varepsilon\right), \varepsilon+i\left(-t_{0}+2 \varepsilon\right)\right)$ for $\varepsilon>0$ small enough. The equivalence relation " $a(\lambda, x, y) \sim b(\lambda, x, y)$ " here means that $a(\lambda, x, y)-b(\lambda, x, y)=O\left(e^{-c \lambda}\right), c>0$. To prove (3.24), it is sufficient to use the estimate $\operatorname{Re} \varphi(p, x, y) \leq-c<0$ for 
$p \in S \backslash \gamma$, which follows from the definition (1.15) for $\varepsilon>0$ small enough. Now (3.20) and (3.22)-(3.24) yield

$$
F(\lambda, x, y) \sim \sum_{j=1}^{2} e^{\lambda A_{j}} \int_{\gamma_{j}^{*}} e^{\lambda\left(-B z+z^{3} / 3\right)} q_{j}(z, \lambda) d z
$$

where $A_{1}=A, A_{2}=\bar{A}$ and

$$
\begin{aligned}
& q_{1}(z, \lambda)=q(p(z, x, y), \lambda, x, y) \frac{\partial}{\partial z} p(z, x, y) \\
& q_{2}(z, \lambda)=q(\overline{p(\bar{z}, x, y)}, \lambda, x, y) \frac{\partial}{\partial z} \overline{p(\bar{z}, x, y)},
\end{aligned}
$$

$\gamma_{j}^{*}$ being the image of the segment $\gamma_{j}$. Notice that $\gamma_{j}^{*} \subset\{z: \operatorname{Re} z>0\}$ and that the end points $\alpha_{j}, \beta_{j}$ of $\gamma_{j}^{*}$ satisfy $\arg \alpha_{j} \in(-\pi / 2,-\pi / 6), \arg \beta_{j} \in(\pi / 6, \pi / 2)$. Further, we use the Weierstrass preparation theorem [2]:

$$
q_{j}(z, \lambda)=r_{j}+\widetilde{r}_{j} z+\left(z^{2}-B\right) \widetilde{q}_{j}(z, \lambda)
$$

and the following representation of the Airy function:

$$
\begin{gathered}
\operatorname{Ai}(s)=\frac{1}{2 \pi i} \int_{\Gamma} e^{-s z+z^{3} / 3} d z, \quad \Gamma=\Gamma_{1} \cup \Gamma_{2}, \quad \text { where } \\
\Gamma_{1}=\left\{z=\varrho \exp \left(i \varphi_{1}\right): \varrho \in(\infty, 0), \varphi_{1} \in(-\pi / 2,-\pi / 6)\right\}, \\
\Gamma_{2}=\left\{z=\varrho \exp \left(i \varphi_{2}\right): \varrho \in(0, \infty), \varphi_{2} \in(\pi / 6, \pi / 2)\right\} .
\end{gathered}
$$

Thus we obtain the uniform asymptotics

$$
F(\lambda, x, y)=\lambda^{-1 / 3} \sum_{k \geq 0}\left(\alpha_{0 k}(\lambda, x, y) \lambda^{-k}+\alpha_{1 k}(\lambda, x, y) \lambda^{-k-1 / 3}\right),
$$

$\lambda \rightarrow \infty$, where the coefficients $\alpha_{j k}$ are given by (1.11). The remainder in the asymptotics (3.25) is estimated as in [1], p. 348.

To verify (1.12), it is sufficient to prove that

$$
|q(p, \lambda, x, y)|+\left|\partial_{x} q(p, \lambda, x, y)\right|+\left|\partial_{p} q(p, \lambda, x, y)\right| \leq c
$$

if $|\operatorname{Re} p| \leq \varepsilon, \varepsilon_{0} \leq|\operatorname{Im} p| \leq \pi / 2-\varepsilon_{0}, \lambda x y \geq 1, x \geq \varepsilon_{0}$ where $\varepsilon_{0}>0$. Since $|\operatorname{Re} z| \geq c>0$ where $z=i \lambda x y / \sinh 2 p$, we can apply the asymptotics of the function $f(z)$ from (3.6), which yields the estimate $\left|f^{(k)}(z)\right| \leq C_{k}|z|^{-k}$. Now (3.26) follows from the definition (3.21). Theorem 5 is proved.

Proof of Theorem 6. We shall use the formulas (3.19) and (3.20), where $\varepsilon=\frac{1}{2} \operatorname{arcosh} x, x^{2} \geq 1+\delta$. The phase function $\varphi$ has critical points $p(x, y)$ and $\bar{p}(x, y)$, where $p(x, y)=\varepsilon+i t, \cos 2 t=y, 0<t<\pi / 2$. They are nondegenerate and $\operatorname{Re} \varphi(p, x, y)<\operatorname{Re} \varphi(p(x, y), x, y)$ if $0 \leq \operatorname{Im} p \leq \pi / 2, p \neq p(x, y), p \in S$, and $\operatorname{Re} \varphi(p, x, y)<\operatorname{Re} \varphi(\bar{p}(x, y), x, y)$ if $-\pi / 2 \leq \operatorname{Im} p \leq 0, p \neq \bar{p}(x, y), p \in S$. 
Applying the saddle-point method [1], we get the asymptotics

$$
F(\lambda, x, y)=\sum_{k \geq 0} a_{k}(\lambda, x, y) \lambda^{-1 / 2-k}, \quad \lambda \rightarrow \infty,
$$

where

$$
\left|a_{k}(\lambda, x, y)\right| \leq c_{k} e^{\lambda \operatorname{Re} \varphi(p(x, y), x, y)}\left(x^{2}-1\right)^{-1 / 4} .
$$

Since $\operatorname{Re} \varphi(p(x, y), x, y)=\frac{1}{2}\left(\operatorname{arcosh} x-x \sqrt{x^{2}-1}\right)$ and

$$
x \sqrt{x^{2}-1}-\operatorname{arcosh} x \geq \delta \sqrt{x^{2}-1} \quad \text { if } x^{2}-1>\delta, 0<\delta<1,
$$

we obtain from $(3.27)-(3.29)$ the estimate

$$
|F(\lambda, x, y)| \leq c \lambda^{-1 / 2} \exp \left(-\frac{1}{2} \lambda \delta \sqrt{x^{2}-1}\right)\left(x^{2}-1\right)^{-1 / 4} .
$$

Now, Theorem 6 follows from (3.30) and (3.19).

To prove Corollary 3 , it is sufficient to obtain the estimate

$$
\begin{aligned}
|F(\lambda, x, y)| \leq c \lambda^{-1 / 3} \exp \left(-c \lambda^{1 / 3}\left(x^{2}-1\right)^{1 / 2}\right) & \\
& \text { if } x^{2}>1+\lambda^{-2 / 3+\varepsilon}, \varepsilon>0, a_{2} \leq \sqrt{\lambda} y \leq A .
\end{aligned}
$$

If $x^{2}>1+\delta$, (3.31) follows from (3.30). Let now $\lambda^{-2 / 3+\varepsilon}<x^{2}-1<\delta, \varepsilon>0$. Then we can apply Theorem 5 . Using the asymptotics of the Airy function and the properties (1.13)-(1.15), (1.17), (1.18), we obtain the asymptotics (3.27) with (3.28). Hence we have again (3.30) with $\delta=\lambda^{-2 / 3+\varepsilon}$, and (3.31) follows.

\section{References}

[1] M. Fedoryuk, The Saddle-Point Method, Nauka, Moscow 1977 (in Russian).

[2] L. Hörmander, The Analysis of Linear Partial Differential Operators I, Springer, 1983.

[3] G. Szegö, Orthogonal Polynomials, Amer. Math. Soc. Colloq. Publ. 23, 1959.

[4] G. Watson, A Treatise on the Theory of Bessel Functions, Cambridge University Press, 1966. 